\title{
The Relation between Platelet/Lymphocyte Ratio and the Occurrence of No Reflow in Patients with ST-Segment Elevation Myocardial Infarction Managed by Primary Percutaneous Coronary Intervention
}

\author{
Ahmed Mohamed El Missiri, Mohamed Rashad Awad, Sameh Maamoun Shaheen \\ Department of Cardiology, Ain Shams University, Cairo, Egypt \\ ORCID: \\ Ahmed Mohamed El Missiri: https://orcid.org/0000-0003-4206-1760 \\ Mohamed Rashad Awad: https://orcid.org/0000-0002-9541-9095 \\ Sameh Maamoun Shaheen: https://orcid.org/0000-0002-1092-3496
}

\section{Abstract}

Introduction: No reflow phenomenon following primary percutaneous coronary intervention (PCI) is a strong predictor of mortality. Platelet/ lymphocyte ratio (PLR) is an indicator of long-term outcome in ischemic heart disease patients. The aim of this study was to assess the relation between PLR measured on admission and the occurrence of no-reflow phenomenon in patients presenting with acute ST-segment elevation myocardial infarction (STEMI) managed by primary PCI. Methods: This was a prospective study including 100 patients with acute STEMI managed by primary PCI. Venous blood samples were obtained on admission to assess hemoglobin level, platelet count, and lymphocyte count. Thrombolysis in myocardial infarction (TIMI) flow grade, myocardial blush grade (MBG), and TIMI thrombus scale were assessed immediately following revascularization. During hospital stay, peak creatinine kinase MB fraction (CK-MB) was recorded, and transthoracic echocardiography was performed to assess left ventricular ejection fraction (LVEF). Results: Patients were divided into two groups based on the TIMI flow grade following PCI: Normal coronary flow group (TIMI 3 flow grade, $n=71$ ) and reduced coronary flow (no-reflow) group (TIMI 0,1 , and 2, $n=29$ ). There was a larger proportion of diabetic patients in the no-reflow group $(P=0.028)$. In addition, patients in the no-reflow group had a more advanced Killip class on presentation $(P=0.001)$, a lower LVEF $(P<0.0001)$, and a significantly higher PLR $213.66 \pm 115.35$ versus $122.81 \pm 59.82(P<0.0001)$. PLR was significantly higher in patients with lower TIMI flow grade and lower MBG $(P<0.0001$ for both). A significant correlation existed generally between PLR and peak CK-MB more in the no-reflow group $(\mathrm{r}=0.471, P=0.01)$. A PLR more than 108.08 predicted no-reflow with a sensitivity of $53 \%$, a specificity of $86 \%$, PPV of $80.5 \%$, and a NPV of $43.1 \%($ AUC $=0.73)$. PLR was found to be an independent predictor of no-reflow multivariate regression analysis for predictors of no-reflow $(B=0.0023,95 \% \mathrm{CI}=0.0014-0.0032, P<0.0001)$. Conclusions: PLR measured on admission is elevated in patients with STEMI who develop no-reflow during primary PCI. PLR is an independent predictor of no-reflow in such patients.

Keywords: Lymphocyte, myocardial infarction, no-reflow, percutaneous coronary intervention, platelet, primary

\section{INTRODUCTION}

Acute myocardial infarction is the leading cause of morbidity and mortality worldwide. ST-segment elevation myocardial infarction (STEMI) carries potentially serious consequences to atherosclerotic coronary artery disease patients. Primary percutaneous coronary intervention (PCI) is the current treatment of choice for patients presenting with STEMI. ${ }^{[1-3]}$

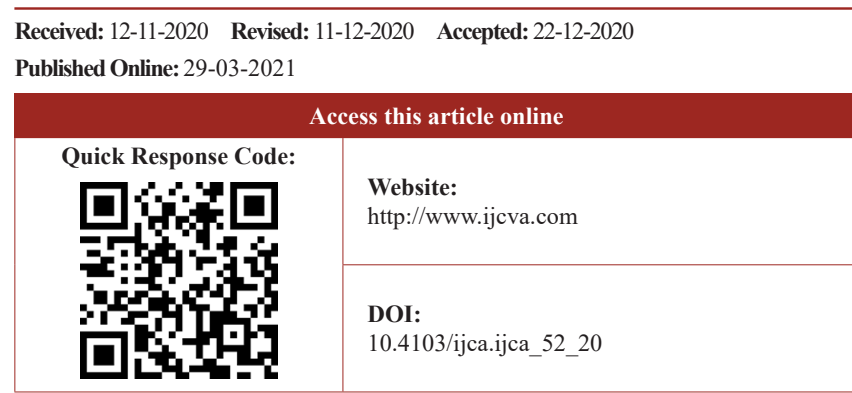

No reflow means the occurrence of myocardial hypoperfusion in the presence of patent coronary arteries. The development of

Address for correspondence: Dr. Ahmed Mohamed El Missiri, Department of Cardiology, Faculty of Medicine, Ain Shams University, Abbassia Square, Abbasia 11566, Cairo, Egypt. E-mail: amissiri@med.asu.edu.eg

This is an open access journal, and articles are distributed under the terms of the Creative Commons Attribution-NonCommercial-ShareAlike 4.0 License, which allows others to remix, tweak, and build upon the work non-commercially, as long as appropriate credit is given and the new creations are licensed under the identical terms.

For reprints contact: WKHLRPMedknow_reprints@wolterskluwer.com

How to cite this article: El Missiri AM, Awad MR, Shaheen SM. The relation between platelet/lymphocyte ratio and the occurrence of no reflow in patients with ST-segment elevation myocardial infarction managed by primary percutaneous coronary intervention. Int J Cardiovasc Acad 2021;7:2-8 
no-reflow during primary PCI is a strong predictor of mortality and carries a negative prognosis irrespective of infarct size. ${ }^{[4,5]}$ Early no-reflow during primary PCI is associated with worse baseline clinical and angiographic characteristics and a worse long-term prognosis. ${ }^{[4-7]}$

Inflammation is a part of the atherosclerotic process. The precipitation of activated platelets leads to the secretion of inflammatory substances from endothelial cells and leukocytes. This leads to monocyte adhesion and migration, thereby exaggerating the inflammatory process and leading to the progression of atherosclerosis. Lymphocytes are also part of the inflammatory process of atherosclerosis, with a lower lymphocyte count associated with increased cardiovascular risk and mortality in STEMI patients ${ }^{[8,9]}$ Platelet/lymphocyte ratio (PLR) has been studied as a marker of long-term outcomes in patients with connective tissue disease and oncologic diseases. Studies have suggested that an elevated PLR might be associated with adverse outcomes in patients with coronary artery disease. ${ }^{[10-12]}$

The aim of this study was to assess the relation between PLR measured on admission and the occurrence of no-reflow after primary PCI for patients presenting with acute STEMI.

\section{Methods}

This was a prospective study performed on 100 consecutive patients presenting to the emergency room at our institution with their first attack of acute STEMI within $12 \mathrm{~h}$ from symptom onset in the period from August 2018 to May 2019. All patients underwent primary PCI as the standard of care provided for such patients. Approval of the Institutional Ethical Committee was obtained for the study protocol, and informed consent was obtained from all patients.

Myocardial infarction was defined using the fourth universal definition of MI as the detection of a rise and/or fall of cardiac biomarkers, with at least one of the values being elevated $\left(>99^{\text {th }}\right.$ percentile upper reference limit) (using creatine kinase [CK]-MB in this study) in addition to symptoms of myocardial ischemia in the past $24 \mathrm{~h}$ and/or new (or presumably new) significant ST-segment elevation or left bundle branch block. ${ }^{[13]}$

Patients were excluded from the study if they had any of the following conditions that represent a possible contraindication for primary PCI; conditions that affect bleeding/coagulation profile; and other conditions that might affect platelet and lymphocyte count: Presenting $>12 \mathrm{~h}$ after the onset on angina or angina equivalent symptoms; presenting with non-ST segment elevation acute coronary syndrome; history of prior myocardial infarction; history of prior PCI or coronary artery bypass grafting; receiving thrombolytic therapy for the index STEMI; recent infection; known hematological abnormalities; history of renal impairment or chronic kidney disease; history of valvular surgery; history of deep venous thromboembolism; receiving chronic anticoagulation for any indication.

\section{Emergency room management}

After confirming the diagnosis and performing a rapid history taking and clinical examination, all patients received loading doses of aspirin (300 mg PO) and clopidogrel (600 mg PO) in the emergency department before being transferred to the cardiac catheterization laboratory. Patients' Killip and Kimball class $^{[14]}$ at presentation was assessed. Admission venous blood samples were obtained from an antecubital puncture to estimate hemoglobin level, platelet count, lymphocyte count, and serum creatinine. PLR was calculated by dividing the absolute platelet count by the absolute lymphocyte count.

\section{Coronary angiography and intervention}

Coronary angiography was performed through a right femoral artery approach in all patients starting with angiography of the non-culprit vessel using a diagnostic catheter after which the culprit vessel was cannulated using a guiding catheter with immediate evaluation of the site of stenosis/occlusion. Each coronary artery was evaluated in several orthogonal views.

Patients underwent primary PCI according to current standards with final stenting using drug-eluting stents. Balloon angioplasty (PTCA) with an undersized balloon was allowed only once per patient to be inflated at its nominal pressure. If a patient required repeated balloon inflations, they were excluded from the study. Post-deployment PTCA with noncompliant balloons was performed as required. Proper stent deployment was confirmed by stent boost technology ${ }^{[15]}$ Glycoprotein $\mathrm{IIb} / \mathrm{III}$ antagonists were not administered before or during the procedure.

Thrombolysis in myocardial infarction (TIMI) flow grade ${ }^{[16]}$ was assessed immediately after stent deployment and before post-deployment PTCA (if performed). Myocardial blush grade $(\mathrm{MBG})^{[17]}$ and TIMI thrombus scale ${ }^{[18]}$ were also assessed.

Final TIMI flow grade ${ }^{[16]}$ was defined as described in published literature into Grade 0 (no perfusion), Grade 1 (penetration without perfusion), Grade 2 (partial perfusion), and Grade 3 (complete perfusion).

$\mathrm{MBG}^{[17]}$ was classified as described in the literature into MBG score 0 (no contrast opacification in the myocardium), MBG score 1 (minimal opacification or persistent staining without washout), MBG score 2 (reduced blush compared to the contralateral noninvolved vessel), and MBG score 3 (normal entry and exit of dye).

The TIMI thrombus scale ${ }^{[18]}$ was classified as described in literature into Grade 0 (no thrombus), Grade 1 (reduced contrast density, haziness, irregular lesion contour, or a smooth meniscus at the site of occlusion), Grade 2 (definite thrombus with its greatest dimension less than half of the vessel diameter), Grade 3 (definite thrombus with its greatest dimension more than half to less than twice the vessel diameter), Grade 4 (definite large thrombus with its greatest 
dimension more than twice the vessel diameter), and Grade 5 (definite complete occlusion of a vessel by a thrombus).

No reflow was defined as having a final TIMI flow Grade of 0 , 1 , or 2 which indicates a failure of reperfusion and has a worse prognosis compared to those with final TIMI flow Grade 3. ${ }^{[19-21]}$

\section{Further laboratory tests and trans-thoracic echocardiography}

During hospital stay, the peak creatinine kinase MB fraction (CK-MB) was recorded as a marker of the infarct size.

Standard transthoracic echocardiography was performed for all patients by an experienced echocardiographer within $48 \mathrm{~h}$ after primary PCI using a Vivid S5 machine with an M4S matrix array probe (General Electric Vingmed, Horten, Norway) to assess left ventricular ejection fraction (LVEF) using the modified Simpson's method of discs. ${ }^{[22]}$

\section{Statistics}

Data were coded, tabulated, and statistically analyzed using Graph Pad Prism (version 8, GraphPad Software, San Diego, CA, USA). The sample size was estimated by the institutional research review committee giving a study power of 80 . Descriptive statistics were done for continuous variables as mean \pm standard deviation, whereas they were done for categorical variables as number and percentage. Tests performed were a two-tailed Student's $t$-test, Fisher's exact test, Pearson's correlation coefficient, and one-way ANOVA test. A receiver-operating characteristic (ROC) curve analysis was used to determine the cutoff level for PLR that predicts no-reflow. Multivariate regression analysis for predictors of no-reflow was performed. The level of significance was taken at a $P<0.05$.

\section{RESULTS}

Patients were divided into two groups based on their TIMI flow grade immediately following PCI: Normal coronary flow group (included those with TIMI 3 flow grade, $n=71$ ) and reduced coronary flow (no-reflow) group (which included those with TIMI flow Grades 0, 1, and 2, $n=29$ ).

\section{Comparing clinical characteristics of both groups}

The no-reflow group had a larger proportion of diabetic patients $18(62.1 \%)$ versus $27(38 \%)(P=0.028)$. There was no difference between both groups regarding age, gender distribution, smoking status, presence of hypertension, and dyslipidemia [Table 1].

In addition, the no-reflow group had a larger proportion of patients in Killip classes 2, 3, and 4 at presentation compared to a larger proportion in Killip class 1 in the normal coronary perfusion group $(P=0.001)$.

\section{Comparing laboratory and echocardiographic findings of both groups}

Patients in the no-reflow group had a higher platelet count $340.24 \pm 122.35$ versus $255.06 \pm 97.59 \times 10^{9} / \mathrm{L}(P<0.0001)$, a lower lymphocyte count $1.80 \pm 0.55$ versus $2.31 \pm 0.85 \times 10^{9} / \mathrm{L}(P=0.004)$, and accordingly a higher PLR $213.66 \pm 115.35$ versus $122.81 \pm 59.82(P<0.0001)$. There was no difference between both groups regarding baseline serum creatinine, baseline hemoglobin levels, and peak CK-MB [Table 1].

In addition, those in the no-reflow group had a lower LVEF of $39.7 \pm 9.5$ versus $47.5 \pm 7.7 \%(P<0.0001)$.

Table 1: Comparing clinical, laboratory, and echocardiographic findings in both groups

\begin{tabular}{|c|c|c|c|}
\hline Variable & Normal coronary flow group $(n=71), n(\%)$ & No reflow group $(n=29), n(\%)$ & $P$ \\
\hline \multicolumn{4}{|l|}{ Clinical characteristics } \\
\hline Age, years & $51.99 \pm 10.29$ & $54.24 \pm 9.07$ & 0.306 \\
\hline Male gender & $59(83.1)$ & $25(86.2)$ & 0.7 \\
\hline Current smoking & $39(54.9)$ & $17(58.6)$ & 0.736 \\
\hline Hypertension & $45(63.4)$ & $19(65.5)$ & 0.84 \\
\hline Type 2 diabetes mellitus & $27(38)$ & $18(62.1)$ & 0.028 \\
\hline Dyslipidemia & $18(25.4)$ & $5(17.2)$ & 0.382 \\
\hline Killip class 1 & $69(97.2)$ & $21(72.4)$ & 0.001 \\
\hline Killip class 2 & $1(1.4)$ & $4(13.8)$ & \\
\hline Killip class 3 & $0(0)$ & $3(10.3)$ & \\
\hline Killip class 4 & $1(1.4)$ & $1(3.4)$ & \\
\hline \multicolumn{4}{|l|}{ Laboratory findings } \\
\hline Peak CK-MB, mg/dl & $348.06 \pm 175.50$ & $386.45 \pm 184.49$ & 0.33 \\
\hline Serum creatinine, $\mathrm{mg} / \mathrm{dl}$ & $1.06 \pm 0.33$ & $1.01 \pm 0.21$ & 0.484 \\
\hline Hemoglobin level, g/dl & $13.12 \pm 1.86$ & $13.02 \pm 2.10$ & 0.818 \\
\hline Platelet count, $\times 10^{9} / \mathrm{L}$ & $255.06 \pm 97.59$ & $340.24 \pm 122.35$ & $<0.0001$ \\
\hline Lymphocyte count, $\times 10^{9} / \mathrm{L}$ & $2.31 \pm 0.85$ & $1.80 \pm 0.55$ & 0.004 \\
\hline Platelet/lymphocyte ratio & $122.81 \pm 59.82$ & $213.66 \pm 115.35$ & $<0.0001$ \\
\hline \multicolumn{4}{|l|}{ Echocardiographic findings } \\
\hline LVEF, $\%$ & $47.5 \pm 7.7$ & $39.7 \pm 9.5$ & $<0.0001$ \\
\hline
\end{tabular}

$\mathrm{LVEF}=$ Left ventricular ejection fraction; $\mathrm{SD}=$ Standard deviation 


\section{Comparing angiographic and procedural findings of both groups}

As would be expected, the normal coronary flow group had a larger proportion of patients with MBG 3, 2, and 1 with all patients in the no-reflow group having an MBG of $0(P<0.0001)$. There was no difference between both groups regarding the frequency of affection of one coronary artery over the other and TIMI thrombus scale [Table 2].

\section{Platelet/lymphocyte ratio}

On further examining PLR for the whole cohort, PLR was significantly higher the lower the TIMI flow grade $(P<0.0001$ for trend) so that it was the highest in those with TIMI 0 flow grade and lowest in those with TIMI 3 flow grade [Table 3].

Similarly, PLR was significantly higher the lower the MBG $(P<0.0001$ for trend $)$ so that it was the highest in those with MBG 0 and lowest in those with MBG 3 [Table 3].
Conversely, PLR was significantly lower the lower the Killip class at presentation $(P<0.001$ for trend) so that it was the highest in those with Killip class 4 and lowest in those with Killip class 1 [Table 3 ].

PLR showed a positive correlation with peak CK-MB levels (reflecting infarct size) $(r=0.312, P=0.002$ for the whole cohort, $r=0.471, P=0.01$ in the no-reflow group).

A PLR of $>108.08$ was found to predict the occurrence of procedural no-reflow in such patients with a sensitivity of $53 \%$, a specificity of $86 \%$, a positive predictive value of $80.5 \%$, and a negative predictive value of $43.1 \%$ (Area under the ROC curve $=0.73)$ [Figure 1].

On performing multivariate regression analysis for predictors of no-reflow after adjustment for variables [Table 4], it was found that PLR was an independent predictor of no-reflow $(ß=0.0023,95 \%$ CI $=0.0014--0.0032$, $P<0.0001)$.

\begin{tabular}{|c|c|c|c|}
\hline Variable & Normal coronary flow group $(n=71), n(\%)$ & No reflow group $(n=29), n(\%)$ & $P$ \\
\hline MBG 3 & $41(57.75)$ & $0(0)$ & $<0.0001$ \\
\hline MBG 2 & $29(40.85)$ & $0(0)$ & \\
\hline MBG 1 & $1(1.4)$ & $0(0)$ & \\
\hline MBG 0 & $0(0)$ & $29(100)$ & \\
\hline TIMI Thrombus scale 1 & $1(1.4)$ & $0(0.0)$ & 0.253 \\
\hline TIMI Thrombus scale 2 & $3(4.2)$ & $0(0.0)$ & \\
\hline TIMI Thrombus scale 3 & $7(9.9)$ & $0(0.0)$ & \\
\hline TIMI Thrombus scale 4 & $1(1.4)$ & $1(3.4)$ & \\
\hline TIMI Thrombus scale 5 & $59(83.1)$ & $28(96.6)$ & \\
\hline \multicolumn{4}{|l|}{ Diseased coronary vessel } \\
\hline Left main coronary artery & $0(0)$ & $0(0)$ & 0.334 \\
\hline LAD only & $37(52.10)$ & $19(65.5)$ & \\
\hline LAD and LCX & $2(2.80)$ & $0(0.00)$ & \\
\hline LAD and RCA & $2(2.80)$ & $2(6.90)$ & \\
\hline LCX only & $10(14.10)$ & $1(3.40)$ & \\
\hline RCA only & $20(28.20)$ & $7(24.10)$ & \\
\hline
\end{tabular}

Categorical variables are expressed as numbers (percentage). $\mathrm{MBG}=$ Myocardial blush grade; TIMI=Thrombolysis in myocardial infarction; $\mathrm{LAD}=\mathrm{Left}$ anterior descending coronary artery; LCX=Left circumflex coronary artery; RCA=Right coronary artery

Table 3: Platelet/lymphocyte ratio in different thrombolysis in myocardial infarction flow grades and myocardial blush grades

\begin{tabular}{|c|c|c|c|c|}
\hline \multicolumn{5}{|c|}{ PLR in relation to different TIMI flow grades } \\
\hline Grade 0 & Grade 1 & Grade 2 & Grade 3 & $P$ \\
\hline $323.33 \pm 14.14$ & $223.21 \pm 87.13$ & $199.34 \pm 125.24$ & $122.81 \pm 59.82$ & $<0.0001$ \\
\hline \multicolumn{5}{|c|}{ PLR in relation to different myocardial blush grades } \\
\hline Grade 0 & Grade 1 & Grade 2 & Grade 3 & $P$ \\
\hline $213.66 \pm 115.35$ & $172.00 \pm 0$ & $150.45 \pm 69.94$ & $102.06 \pm 42.23$ & $<0.0001$ \\
\hline \multicolumn{5}{|c|}{ PLR in relation to Killip class at presentation } \\
\hline Killip 1 & Killip 2 & Killip 3 & Killip 4 & $P$ \\
\hline $131.52 \pm 66.34$ & $255.12 \pm 100.09$ & $378.25 \pm 105.51$ & $334.09 \pm 170.35$ & $<0.001$ \\
\hline
\end{tabular}

Continuous variables are expressed as mean and SD. TIMI=Thrombolysis in myocardial infarction; PLR=Platelet-lymphocyte ratio; $\mathrm{SD}=$ Standard deviation 


\begin{tabular}{|c|c|c|c|c|c|c|}
\hline \multirow[t]{2}{*}{ Variable } & \multirow[t]{2}{*}{ В } & \multirow[t]{2}{*}{ SE } & \multirow[t]{2}{*}{$T$} & \multirow[t]{2}{*}{$P$} & \multicolumn{2}{|c|}{$95 \% \mathrm{Cl}$} \\
\hline & & & & & Lower & Upper \\
\hline Age & 0.002 & 0.004 & 0.609 & 0.544 & -0.005 & 0.010 \\
\hline Gender & 0.028 & 0.121 & 0.231 & 0.818 & -0.213 & 0.269 \\
\hline Smoking status & -0.030 & 0.089 & 0.339 & 0.339 & -0.209 & 0.148 \\
\hline Hypertension & -0.079 & 0.089 & 0.890 & 0.376 & -0.257 & 0.098 \\
\hline Diabetes mellitus & 0.118 & 0.084 & 1.415 & 0.161 & -0.048 & 0.285 \\
\hline Dyslipidemia & -0.099 & 0.091 & 1.094 & 0.277 & -0.281 & 0.082 \\
\hline Peak CK-MB level & 0.0001 & 0.0002 & 0.430 & 0.668 & -0.0004 & 0.0006 \\
\hline Platelet/lymphocyte ratio & 0.0023 & 0.0004 & 4.902 & $<0.0001$ & 0.0014 & 0.0032 \\
\hline
\end{tabular}

$\mathrm{CI}=$ Confidence interval; $\mathrm{SE}=$ Standard error; $\mathrm{CK}-\mathrm{MB}=$ Creatine kinase-MB fraction

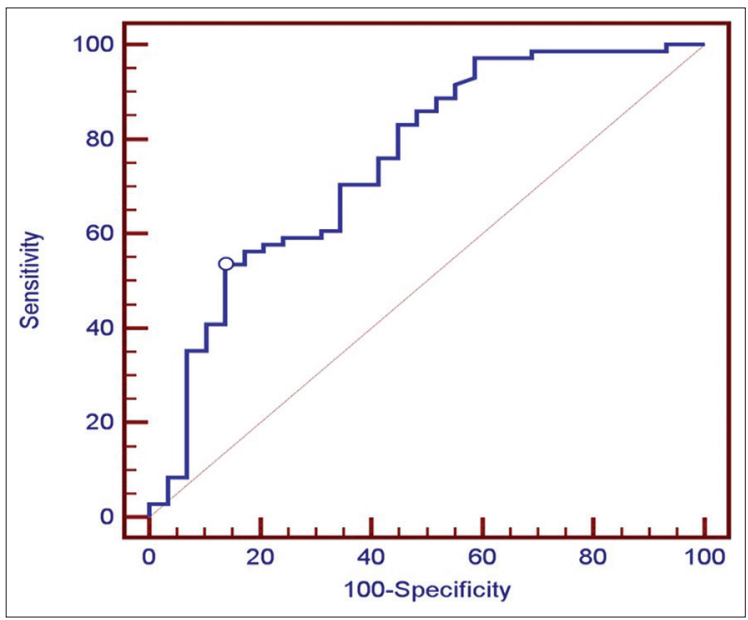

Figure 1: Receiver operating characteristic curve analysis of platelet/ lymphocyte ratio for occurrence of no reflow

\section{Discussion}

The main findings of this study are that (1) PLR was significantly elevated in patients presenting with acute STEMI managed by primary PCI who develop no-reflow during the procedure compared to those with normal coronary perfusion; (2) PLR was an independent predictor of no-flow in such patients; (3) PLR was higher the lower the final TIMI flow grade; (4) PLR was higher the lower the MBG; (5) PLR more than 108.08 predicted the occurrence of no-reflow in such patients with good specificity but relatively weak sensitivity; (6) PLR was higher the higher Killip class at presentation.

The secondary findings included that the incidence of no-reflow in this study was $29 \%$. Those who developed no-reflow had a more advanced Killip class at presentation, were more frequently diabetic, and had a lower LVEF after the procedure. Our study was a prospective one in contrast to most studies examining the relation of PLR to various conditions in the literature which tend to be retrospective.

PLR has been used as a biomarker in a wide array of diseases and conditions that combine pro-thrombotic states with acute inflammation. It has been studied in cancer patients, systemic lupus erythematosus, Behcet's disease, thyroid disease, pulmonary embolism, and even mood disorders. Its increase is generally associated with worse outcomes, disease activity, and poor survival. ${ }^{[23-26]}$

PLR was also studied in patients with different forms of coronary artery disease where in most instances it was associated with a worse prognosis. In patients suffering from acute coronary syndromes, it is considered a predictor of cardiovascular adverse events and all-cause mortality. The same was reported in those with high-risk stable coronary artery disease. ${ }^{[12,27]}$

Studies have shown that in STEMI patients managed by primary PCI, impaired coronary perfusion, and flow at the end of the procedure are associated with poor outcomes in both the short and long term and are associated with increased mortality. ${ }^{[28,29]}$ Researchers have reported that predictors of no-reflow in the setting of primary PCI include diabetes mellitus, impaired LVEF on admission, prolonged pain-to-balloon time, high thrombus burden, and an initial TIMI flow of $\leq 1$. $^{[30-33]}$

Most studies in the literature examining the relation of PLR to various outcomes in coronary artery disease patients are retrospective studies in contrast to our study. In a study on 520 patients with acute STEMI managed by primary PCI, the incidence of no-reflow was $22.5 \%$. PLR measured before intervention was significantly higher in those with no-reflow compared to those with normal coronary perfusion. A preprocedural PLR of more than 126 was predictive of the occurrence of postprocedural no-reflow with a sensitivity of $71 \%$ and a specificity of $73 \% .{ }^{[28]}$ These findings are similar to those of the current study; however, the cutoff value of PLR reported (126) had a higher sensitivity, but lower specificity than the one we report (108.08).

Another study followed a cohort of 514 patients with high-risk coronary artery disease undergoing coronary angiography for different indications for a median follow-up period of 5 years. They divided patients into tertiles based on their preprocedural PLR. They found that those with a PLR of $>145$ had a worse prognosis than those with a PLR of $<106$ and those with a PLR of 106.1-145. A PLR $\geq 137$ was associated with higher all-cause mortality. The authors concluded that PLR may be a useful biomarker to predict the prognosis in such patients. ${ }^{[27]}$ 
Another study was performed on 587 patients with acute coronary syndrome undergoing coronary angiography where patients were divided into tertiles according to their PLR. The authors found that in-hospital mortality was highest in patients in the upper PLR tertile. Using a cutoff value of 142 PLR was found to predict in-hospital mortality with a sensitivity of $69 \%$ and a specificity of $63 \% .{ }^{[30]}$ PLR was also reported to be significantly higher in a group of 78 patients with slow coronary flow on comparing them to 50 patients with normal coronary flow. ${ }^{[34]}$

An earlier study examined patients with normal coronary anatomy on angiography of whom 221 had slow coronary flow (defined in this study as a TIMI frame count $>2$ ) and 293 were considered healthy controls. Similar to the findings of the current study, the authors found that PLR was significantly higher in the slow coronary flow group and through logistic regression analysis PLR was found to be independently associated with the occurrence of coronary slow flow. ${ }^{[35]}$

Additionally, researchers examined the prognostic value of PLR in primary PCI in 440 patients who had acute STEMI. Patients were divided into a low PLR group $(<137)$ and a high PLR group ( $>137)$. Patients in the high PLR group had higher SYNTAX scores, higher mortality, and a higher incidence of no-reflow (defined as a TIMI flow grade other than 3 ). The authors concluded that PLR is an independent risk factor of no-reflow (in agreement with the findings of the current study) and in-hospital adverse events. ${ }^{[36]}$

High PLR was found to be an independent risk factor for the development of inadequate myocardial perfusion in 445 STEMI patients who underwent primary PCI similar to the results of the current study. High PLR patients $(\geq 165.33$ ) more frequently had TIMI flow grades $0-2$ and MPG $0-1$. These patients also had more advanced heart failure and in-hospital major adverse cardiac events. ${ }^{[37]}$

\section{Study limitations}

The limitations of the current study are that it was performed in a single medical center with a relatively small number of patients. Results are mainly driven by postprocedural angiographic findings. No long-term follow-up or assessment of major adverse cardiovascular events was performed. Intravascular ultrasound imaging was not performed, and assessment of coronary flow and thrombus burden was only assessed angiographically.

\section{Conclusions}

PLR measured on admission is elevated in patients with STEMI who develop no-reflow during primary PCI. PLR is an independent predictor of no-reflow in such patients.

\section{Financial support and sponsorship}

Nil.

\section{Conflicts of interest}

There are no conflicts of interest.

\section{References}

1. Ibanez B, James S, Agewall S, Antunes MJ, Bucciarelli-Ducci C, Bueno H, et al. 2017 ESC Guidelines for the management of acute myocardial infarction in patients presenting with ST-segment elevation: The Task Force for the management of acute myocardial infarction in patients presenting with ST-segment elevation of the European Society of Cardiology (ESC). Eur Heart J 2018;39:119-77.

2. Levine GN, Bates ER, Blankenship JC, Bailey SR, Bittl JA, Cercek B, et al. 2015 ACC/AHA/SCAI Focused Update on Primary Percutaneous Coronary Intervention for Patients With ST-Elevation Myocardial Infarction: An Update of the 2011 ACCF/AHA/SCAI Guideline for Percutaneous Coronary Intervention and the 2013 ACCF/AHA Guideline for the Management of ST-Elevation Myocardial Infarction: A Report of the American College of Cardiology/American Heart Association Task Force on Clinical Practice Guidelines and the Society for Cardiovascular Angiography and Interventions. Circulation 2016;133:1135-47.

3. O'Gara PT, Kushner FG, Ascheim DD, Casey DE Jr., Chung MK, de Lemos JA, et al. 2013 ACCF/AHA guideline for the management of ST-elevation myocardial infarction: A report of the American College of Cardiology Foundation/American Heart Association Task Force on Practice Guidelines. J Am Coll Cardiol 2013;61:e78-140.

4. Allencherril J, Jneid H, Atar D, Alam M, Levine G, Kloner RA, et al. Pathophysiology, diagnosis, and management of the no-reflow phenomenon. Cardiovasc Drugs Ther 2019;33:589-97.

5. Jiecheng $\mathrm{P}, \mathrm{Ai}$-Ling W. Clinical significance of no-reflow in different stages of primary angioplasty among patients with acute myocardial infarctions. Perfusion 2016;31:300-6.

6. Rezkalla SH, Stankowski RV, Hanna J, Kloner RA. Management of No-Reflow Phenomenon in the Catheterization Laboratory. JACC Cardiovasc Interv 2017;10:215-23.

7. Jaffe R, Charron T, Puley G, Dick A, Strauss BH. Microvascular obstruction and the no-reflow phenomenon after percutaneous coronary intervention. Circulation 2008;117:3152-6.

8. Ommen SR, Gibbons RJ, Hodge DO, Thomson SP. Usefulness of the lymphocyte concentration as a prognostic marker in coronary artery disease. Am J Cardiol 1997;79:812-4.

9. Sarma J, Laan CA, Alam S, Jha A, Fox KA, Dransfield I. Increased platelet binding to circulating monocytes in acute coronary syndromes. Circulation 2002;105:2166-71.

10. Akkaya E, Gul M, Ugur M. Platelet to lymphocyte ratio: A simple and valuable prognostic marker for acute coronary syndrome. Int J Cardiol 2014;177:597-8.

11. Bressi E, Mangiacapra F, Ricottini E, Cavallari I, Colaiori I, Di Gioia $\mathrm{G}$, et al. Impact of neutrophil-to-lymphocyte ratio and platelet-to-lymphocyte ratio on 5-Year clinical outcomes of patients with stable coronary artery disease undergoing elective percutaneous coronary intervention. J Cardiovasc Transl Res 2018;11:517-23.

12. Li H, Zhou Y, Ma Y, Han S, Zhou L. The prognostic value of the platelet-to-lymphocyte ratio in acute coronary syndrome: A systematic review and meta-analysis. Kardiol Pol 2017;75:666-73.

13. Thygesen K, Alpert JS, Jaffe AS, Chaitman BR, Bax JJ, Morrow DA, et al. Fourth universal definition of myocardial infarction (2018). J Am Coll Cardiol 2018;72:2231-64.

14. Killip $\mathrm{T} 3^{\text {rd }}$, Kimball JT. Treatment of myocardial infarction in a coronary care unit. A two year experience with 250 patients. Am J Cardiol 1967;20:457-64.

15. Tanaka N, Pijls NH, Koolen JJ, Botman KJ, Michels HR, Brueren BR, et al. Assessment of optimum stent deployment by stent boost imaging: Comparison with intravascular ultrasound. Heart Vessels 2013;28:1-6.

16. Group TS. The thrombolysis in myocardial infarction (TIMI) trial. Phase I findings. N Engl J Med 1985;312:932-6.

17. van 't Hof AW, Liem A, Suryapranata H, Hoorntje JC, de Boer MJ, Zijlstra F. Angiographic assessment of myocardial reperfusion in patients treated with primary angioplasty for acute myocardial infarction: Myocardial blush grade. Zwolle Myocardial Infarction Study Group. Circulation 1998;97:2302-6.

18. Gibson CM, de Lemos JA, Murphy SA, Marble SJ, McCabe CH, Cannon $\mathrm{CP}$, et al. Combination therapy with abciximab reduces angiographically evident thrombus in acute myocardial infarction: 
A TIMI 14 substudy. Circulation 2001;103:2550-4.

19. Eeckhout E, Kern MJ. The coronary no-reflow phenomenon: A review of mechanisms and therapies. Eur Heart J 2001;22:729-39.

20. Ramjane K, Han L, Jin C. The diagnosis and treatment of the no-reflow phenomenon in patients with myocardial infarction undergoing percutaneous coronary intervention. Exp Clin Cardiol 2008;13:121-8.

21. Simes RJ, Topol EJ, Holmes DR Jr., White HD, Rutsch WR, Vahanian A, et al. Link between the angiographic substudy and mortality outcomes in a large randomized trial of myocardial reperfusion. Importance of early and complete infarct artery reperfusion. GUSTO-I Investigators. Circulation 1995;91:1923-8.

22. Lang RM, Bierig M, Devereux RB, Flachskampf FA, Foster E, Pellikka PA, et al. Recommendations for chamber quantification: A report from the American Society of Echocardiography's Guidelines and Standards Committee and the Chamber Quantification Writing Group, developed in conjunction with the European Association of Echocardiography, a branch of the European Society of Cardiology. J Am Soc Echocardiogr 2005;18:1440-63.

23. Gasparyan AY, Ayvazyan L, Mukanova U, Yessirkepov M, Kitas GD. The platelet-to-lymphocyte ratio as an inflammatory marker in rheumatic diseases. Ann Lab Med 2019;39:345-57.

24. Li B, Zhou P, Liu Y, Wei H, Yang X, Chen T, et al. Platelet-to-lymphocyte ratio in advanced cancer: Review and meta-analysis. Clin Chim Acta 2018;483:48-56.

25. Mazza MG, Lucchi S, Tringali AG, Rossetti A, Botti ER, Clerici M. Neutrophil/lymphocyte ratio and platelet/lymphocyte ratio in mood disorders: A meta-analysis. Prog Neuropsychopharmacol Biol Psychiatry 2018;84:229-36.

26. Wang Q, Ma J, Jiang Z, Ming L. Prognostic value of neutrophil-to-lymphocyte ratio and platelet-to-lymphocyte ratio in acute pulmonary embolism: A systematic review and meta-analysis. Int Angiol 2018;37:4-11.

27. Lee YS, Baradi A, Peverelle M, Sultani R, Adams H, Garlick J, et al. Usefulness of platelet-to-lymphocyte ratio to predict long-term all-cause mortality in patients at high risk of coronary artery disease who underwent coronary angiography. Am J Cardiol 2018;121:1021-6.

28. Kurtul A, Yarlioglues M, Murat SN, Ergun G, Duran M, Kasapkara HA, et al. Usefulness of the platelet-to-lymphocyte ratio in predicting angiographic reflow after primary percutaneous coronary intervention in patients with acute ST-segment elevation myocardial infarction. Am J Cardiol 2014;114:342-7.

29. Stone GW, Peterson MA, Lansky AJ, Dangas G, Mehran R, Leon MB. Impact of normalized myocardial perfusion after successful angioplasty in acute myocardial infarction. J Am Coll Cardiol 2002;39:591-7.

30. Nair Rajesh G, Jayaprasad N, Madhavan S, Sudha Kumary V, Jayaprakash K, Raihanathul Misiriya KJ, et al. Predictors and prognosis of no-reflow during primary percutaneous coronary intervention. Proc (Bayl Univ Med Cent) 2019;32:30-3.

31. Fajar JK, Heriansyah T, Rohman MS. The predictors of no reflow phenomenon after percutaneous coronary intervention in patients with ST elevation myocardial infarction: A meta-analysis. Indian Heart $\mathrm{J}$ 2018;70 Suppl 3:S406-18.

32. Avci E, Yildirim T, Aydin G, Kiris T, Dolapoglu A, Kadi H, et al. Combining clinical predictors to better predict for the no-reflow phenomenon. Eur Rev Med Pharmacol Sci 2018;22:4987-94.

33. Oylumlu M, Yıldız A, Oylumlu M, Yüksel M, Polat N, Bilik MZ, et al. Platelet-to-lymphocyte ratio is a predictor of in-hospital mortality patients with acute coronary syndrome. Anatol J Cardiol 2015;15:277-83.

34. Çetin M, Kiziltunc E, Elalmış ÖU, Çetin ZG, Demirçelik MB, Çiçekçioğlu $\mathrm{H}$, et al. Predictive value of neutrophil lymphocyte ratio and platelet lymphocyte ratio in patients with coronary slow flow. Acta Cardiol Sin 2016;32:307-12.

35. Akboga MK, Canpolat U, Balci KG, Akyel A, Sen F, Yayla C, et al. Increased Platelet to Lymphocyte Ratio is Related to Slow Coronary Flow. Angiology 2016;67:21-6.

36. Ayca B, Akin F, Okuyan E. Platelet to lymphocyte ratio as a prognostic marker in primary percutaneous coronary intervention. Platelets 2015;26:816.

37. Maimaiti A, Li Y, Wang YT, Yang X, Li XM, Yang YN, et al. Association of platelet-to-lymphocyte count ratio with myocardial reperfusion and major adverse events in patients with acute myocardial infarction: A two-centre retrospective cohort study. BMJ Open 2019;9:e025628 